\title{
El nuevo marco de la política regional española tras la integración en la CEE
}

\author{
M. a Victoria Azcárate luxán, Carmen Muguruza Cañas y \\ José Miguel Santos Preciado
}

\section{INTRODUCCION}

En el presente articulo se recoge la ponencia sobre «la política regional comunitaria" que tuvo lugar en el Seminario sobre "Las Consecuencias geográficas de la entrada de España en la CEE», dicha ponencia intentaba esbozar el estado de la cuestión sobre los problemas de los desequilibrios regionales en la Comunidad Económica Europea y los instrumentos de política regional que pretenden paliar dichos desequilibrios.

El acercamiento a la cuestión lo enfocamos en tres grandes apartados:

En primer lugar, analizar cuáles son los nuevos problemas regionales y urbanos que surgen tras la crisis económica en el ámbito europeo y español. Los años de crecimiento económico, entre los cincuenta y setenta, provocaron intensísimos desajustes territoriales y regionales. El desarrollo económico fue industrial y urbano, frente al crecimiento de las grandes y medianas ciudades, se produjo una intensa desertización de amplios espacios regionales, en su mayor parte agrarios, que perdieron población y recursos en favor de las regiones industriales y urbanas. A partir de la crisis económica de los años 70 , que deja sentir sus efectos territoriales a finales de esa década y comienzos de la actual, se provocan nuevos problemas regionales. Con la crisis económica aparecen otros problemas territoriales que se suman a los del pasado. Por ello estimamos necesario conocer y analizar, aunque sea brevemente, la nueva situación territorial de la sociedad postcrisis, pues sólo conociendo y siendo conscientes de los nuevos pro- 
blemas regionales y urbanos se puede establecer una política regional capaz de resolver la nueva situación planteada.

En segundo lugar analizaremos los desequilibrios regionales en la Europa Comunitaria, y los indicadores que sirven para medir dichos desequilibrios, así como la clasificación de las regiones problemáticas, según el III Informe de la Comisión de Desarrollo Regional de la CEE. Centrando el análisis en uno de los más importantes instrumentos de política regional de la Comunidad, el Fondo Europeo para el Desarrollo Regional (FEDER).

Por último abordamos en el artículo la panorámica española. Tras la integración de España en la Comunidad Económica Europea, y tras la crisis económica, surge una nueva problemática regional en nuestro pais. Asimismo hay que tener en cuenta la nueva división territorial del poder, surgida en 1978, tras el establecimiento de la España de las Autonomías, y los nuevos instrumentos existentes para resolver los problemas territoriales, todo ello ha dado como resultado una nueva forma de enfocar los problemas regionales. Finalmente se analiza la nueva política regional existente en España tras la incorporación en la Comunidad, sus exigencias, los instrumentos (en concreto los Planes de Desarrollo Regional) y los problemas que puede acarrear dicha política.

\section{EL MARCO DE LA POLITICA REGIONAL: LOS NUEVOS PROBLEMAS TERRITORIALES}

El crecimiento económico de los años cincuenta y sesenta fue industrial pero sobre todo fue terciario y urbano. Crecieron aceleradamente las ciudades grandes y medianas, incluso en muchos casos no fueron las grandes ciudades las que crecieron sino sus periferias, constituyéndose asi áreas o regiones metropolitanas, verdaderos espacios regionales altamente urbanizados, que fueron la expresión más característica de la expansión económica. Al mismo tiempo que se producia la metropolitanización de los sistemas urbanos europeos, se acentuaban los desequilibrios territoriales y bastantes zonas entraban en un proceso de estancamiento y decadencia, en tanto que en las áreas metropolitanas modernas y dinámicas el crecimiento era pagado con costes sociales a veces muy altos y las periferias subequipadas y marginadas eran la triste expresión del desarrollo. Un tipo de situación consecuente al modelo de concentración de la época del crecimiento económico 
de los cincuenta-sesenta son las zonas del territorio semiabandonadas y la crisis de los pequeños municipios, cuya población, si no emigra de la región, es atraída por la ciudad grande o mediana más próxima. La otra consecuencia es el hecho metropolitano que desborda y sumerge a la gran ciudad, incapaz de gestionarlo y financiarlo.

Los cambios que se han producido en la economía mundial en el último decenio tienen su reflejo en la aparición de nuevos problemas en el ámbito regional y urbano.

En España como en otros paises europeos, la crisis está dando lugar a un acrecentamiento de los problemas territoriales, no sólo por su multiplicación e intensidad, sino porque muchos son distintos, en su naturaleza, a los que conociamos en un pasado reciente.

La ruptura del proceso de crecimiento sostenido que se habia producido en las economías industrializadas hasta comienzos de los años setenta, supone el inicio de una crisis profunda en la economía mundial, lo cual ha inducido a intensos cambios. Estos cambios, han supuesto no sólo problemas económicos globales, sino problemas territoriales específicos.

Con la crisis comienza a surgir en Europa un nuevo «Problema Regional" que se añade y da complejidad a los problemas heredados, los cuales, a su vez, se ven con frecuencia agravados. En este sentido, a las disparidades intrarregionales que constituyeron el problema europeo de los años 60 , se añaden ahora las crecientes disparidades intrametropolitanas o intra urbanas.

La crisis económica de mitad de los setenta tendrá importante efectos sobre el modelo territorial, suponiendo, en parte, la quiebra del modelo de concentración de la población y los recursos en las grandes aglomeraciones urbanas.

Manuel CASTELL (1983) estudiando los efectos territoriales de la crisis ha puesto de manifiesto que la crisis económica sobre el territorio se puede definir por cuatro rasgos principales:

El primer rasgo sería un proceso de reestructuración regional. Es decir, un cambio de sentido de los procesos de concentración de la actividad económica, la población y los recursos que no siguen la ley, que parecia in- 
mutable, de que siempre las regiones más desarrolladas van a acumular las tasas de crecimiento más favorables.

El segundo rasgo sería un descenso en la tasa de crecimiento metropolitano, que a veces puede llegar a ser negativa.

El tercer rasgo es el crecimiento de las ciudades medias y el cuarto rasgo el crecimiento de las zonas rurales, con disminución paralela de las actividades agrícolas.

Sin embargo, todo ello no quiere decir, como señala el profesor Castell, que la era de las grandes metrópolis haya terminado.

Después de la crisis económica una cosa queda clara, han cambiado en parte las tendencias territoriales que dominaron la época de la industrialización acelerada. Es más, nos encontramos en un momento insólito en que se corta un largo proceso de acumulación de la población en torno a las mayores aglomeraciones urbanas. Un proceso de concentración que contaba con siglos de antigüedad.

En este proceso de concentración las áreas metropolitanas constituyeron el lugar espacial del modelo que entra en crisis en la década de los setenta, lo cual ha conducido a que en el momento actual estos espacios acumulen en su interior una gran parte de los problemas más dramáticos de la coyuntura económica-social.

Los efectos más relevantes de la crisis han sido:

En primer lugar, la crisis del sistema industrial de base urbana que ha provocado el hundimiento y desmantelamiento de una buena parte de las estructuras productivas de las áreas metropolitanas. Especialmente las áreas de vieja tradición industrial.

El mayor impacto se ha producido en aquellas áreas cuyos tejidos industriales presentan un alto grado de especialización y una importante presencia de actividades maduras mientras que las áreas con una estructura industrial diversificada y con una presencia significativa de sectores dinámicos, han soportado mejor la crisis. 
En segundo lugar, la crisis ha provocado una crisis social marcada por la caída del empleo. Los efectos sociales de la caida de la base productiva de la ciudad han sido de gran amplitud. La crisis ha producido un fuerte incremento de la segmentación social, consolidando la fragmentación del mercado laboral y fomentando el desarrollo de impresionantes bolsas de mercado de trabajo marginal.

La crisis de la ciudad se define por la marginación y el gheto, por el incremento de la dualidad que enfrenta a parados y ocupados, la economia legal y la economía subterránea, la ciudad del cuaternario y la de la industria marginal, la alta productividad de los sectores avanzados y el paro estructural.

En tercer lugar, la crisis ha producido la degradación del marco urbano. La crisis, en España, se produce en un marco urbano sumamente degradado en todos los aspectos. El proceso de crecimiento metropolitano español, realizado de una forma tardía y acelerada bajo una politica urbana netamente especulativa, ha dado lugar a la existencia de áreas sumamente degradadas y deficitarias en servicios y equipamientos, cuya calidad ambiental ha sufrido niveles altísimos de degradación que refuerzan y agravan la crisis de los espacios urbanos. Areas industriales abandonadas o infrautilizadas, barrios de promoción pública de baja calidad constructiva en proceso casi irreversible de deterioro, degradación de barrios antiguos en los cascos urbanos son algunos de los efectos más visibles de la actual crisis del marco urbano.

En cuarto lugar, la crisis ha favorecido un uso degradante del territorio, produciéndose un incremento de usos marginales del espacio, tales como la difusión de la segunda residencia ilegal, la intensificación de la promoción ilegal de suelo industrial etc...

Pero también existen algunos elementos que pudieran ser positivos, puede que la crisis del modelo de concentración metropolitana no constituya necesariamente el inicio de un proceso degenerativo. De hecho, el modelo de concentración provocó intensísimos desajustes territoriales. La ralentización de los procesos de polarización y concentración puede traducirse a medio plazo en un tipo de desarrollo cualitativo del modelo territorial.

En las tendencias de descentralización de la actividad económica, en la detención de los flujos migratorios, en las inversiones en los espacios menos desarrollados, se encuentra la posibilidad de articular una organización espacial y regional más equilibrada. 
De hecho, la difusión de las actividades productivas sobre el territorio y los procesos de descentralización pueden constituir un elemento de nivelación de las rentas regionales, produciéndose una detención de la degradación demográfica de ciertas áreas.

\section{LA POLITICA REGIONAL COMUNITARIA}

Es un hecho evidente que los desequilibrios regionales de la Comunidad Europea se han ido agudizando con las sucesivas ampliaciones. Si en un principio no existia una excesiva heterogeneidad en la Comunidad de los seis, lógicamente si exceptuamos el Mezzogiorno italiano y alguna que otra región aislada, con el paso del tiempo, al incorporarse el Reino Unido, y sobre todo Irlanda, con unas regiones mucho menos desarrolladas, las disparidades regionales se fueron acentuando.

Más adelante, la incorporación de paises con unos rendimientos económicos muy inferiores a la media comunitaria, con un desempleo superior, con una fuerte presión demográfica, además de poseer una importante proporción de empleos agrícolas y unos niveles internos de desarrollo muy diferentes, supuso una mayor heterogeneidad dentro de la Comunidad.

Así, por ejemplo, con la entrada de España y Portugal el número de personas ocupadas y el volumen de producción aumentó en un 12-13 por 100 , y el número de habitantes en un 18 por 100 , mientras que el número de parados lo hizo en un 30 por 100 y el número de personas ocupadas en el sector agrario en un 36 por 100 (Comisión CEE, 1987, pág. 1). Asimismo, ninguna de las regiones de los dos nuevos Estados miembros supera la renta media comunitaria.

Gracias a las numerosas publicaciones de la Oficina Estadística de las Comunidades Europeas, a las que se añaden aquéllas de los Institutos $\mathrm{Na}$ cionales de Estadística, actualmente se puede conocer con bastante exactitud la magnitud de los desequilibrios regionales que afectan a la Comunidad. En este sentido, son varios los indicadores socioeconómicos que se vienen utilizando para apreciar mejor la intensidad de estos desequilibrios regionales y poder poner en práctica una eficaz politica regional a nivel comunitario. La mayor parte de los estudios realizados hasta ahora emplean la información estadística suministrada por la CEE, fundamen- 
talmente el análisis de la renta y nivel de productividad (exponentes de la estructura económica) y los índices de paro (exponentes de los desequilibrios estructurales en el mercado de trabajo). Ambas variables, referidas a la producción y al empleo, se combinan en un índice sintético que permite obtener una visión más completa sobre la magnitud de los problemas regionales a nivel comunitario, contribuyendo asi a definir las prioridades geográficas de intervención de la política regional comunitaria.

Con la adhesión de España y Portugal, las disparidades productivas regionales, expresadas en el PIB por habitante, lejos de disminuir han alcanzado unos niveles más elevados. Las regiones afectadas, en las que vive casi una quinta parte de la población de la Comunidad, se encuentran situadas en Grecia, Mezzogiorno italiano, sector meridional y occidental de la Península Ibérica, Irlanda y algunas regiones del norte de la Comunidad con problemas específicos. Otro indicador socioeconómico clave como es la productividad de una región, expresada en el PIB por persona activa, arroja un resultado parecido; de nuevo la periferia meridional y occidental de la Comunidad destaca por su baja productividad.

Las disparidades existentes en el paro, a nivel regional en la Comunidad de los doce, han aumentado considerablemente. Globalmente, la tasa de desempleo pasó del 2.5 por 100 en 1973 al 12 por 100 en 1985. Este progresivo crecimiento del paro, que obedece a diferentes factores según las regiones, va acompañado de un crecimiento, en términos absolutos, de los desequilibrios regionales del paro. Por regla general, en este sentido, se puede constatar que las regiones cuyo nivel de producción es más bajo son las que precisamente ostentan unos mayores índices de desempleo: Italia meridional, casi todas las regiones de España, Irlanda y algunas zonas industriales del Reino Unido (zonas con un gran peso industrial durante el siglo xx, pero que hoy día padecen los efectos de la reconversión).

Por otra parte, ciertos colectivos, especialmente las mujeres y los menores de 25 años, sufren el problema del desempleo de una manera desproporcionada. A nivel regional, las regiones más afectadas por las tasas de paro femenino y juvenil se encuentran de nuevo en los sectores meridional y occidental de la Comunidad, asi como en algunas regiones holandesas $y$ belgas.

Asi pues, al evaluar el indice sintético se pone de relieve que las regiones con mayores problemas, es decir, con el paro más elevado y la renta más baja, se encuentran en el área meridional y occidental de la Comunidad. Sintetizando, se trata, por una parte, de regiones con una fuerte orientación agricola 
(Grecia, Mezzogiorno italiano, Córcega, España, Portugal, Irlanda del Norte), y por otra, de algunas regiones industriales (ciertas regiones del Reino Unido, Bélgica, RFA y Francia) que hoy día se enfrentan con problemas específicos de reajuste industrial.

Finalmente, la Comisión de las Comunidades Europeas tiene también en cuenta otros factores que influyen en estos desequilibrios, ya sean por si mismos o por su incidencia sobre la producción y el empleo. En este sentido, resulta muy significativo analizar la densidad demográfica regional, la intensidad de los movimientos migratorios regionales, así como la específica dotación en infraestructuras de las diferentes regiones (Comisión CEE, 1987, págs. 30-35 y anexo).

La clasificación de regiones europeas según sus características socioeconómicas no es ninguna novedad; son muchos los criterios adoptados, y por ello las tipologias regionales difieren según los distintos autores (CONDOMINES, 1982, pág. 430). Las tipologías basadas en una sola variable son muy numerosas; sin embargo, su mayor inconveniente reside tanto en la arbitrariedad de elección de la variable como del grado de jerarquización regional, sin que ningún otro criterio objetivo sea tenido en cuenta. Implícitamente, la mayor parte de los mapas elaborados en las publicaciones oficiales de la Comunidad pertenecen a esta categoria; de hecho, la trama cartográfica de las diferentes regiones corresponde al valor que adquiere una variable en una determinada región en relación a la media de esta variable en el conjunto de las regiones europeas.

Las tipologías basadas en la utilización de varios indicadores reflejan mucho mejor la problemática regional. Entre estas tipologías podemos distinguir aquellas cuya finalidad es poner de manifiesto una determinada estructura económica a partir de distintas variables, y otras que afrontan el estudio de un problema particular utilizando diversos indicadores que giran en torno a esta cuestión.

En este sentido, la Comisión de las Comunidades Europeas establece una tipología que utiliza como instrumento para medir la intensidad relativa de los problemas regionales y que sive, sobre todo, como punto de referencia para fijar las modalidades y la orientación de las medidas que se han de aplicar a cada zona. Así, en función del estado de desarrollo, de la estructura por sectores, del asentamiento de la población y de la distinta situación física de las regiones, la Comisión adopta una tipología basada en seis grandes tipos de utilización frecuente, así como tres subcategorías. Son las regiones atrasadas, regiones industriales en decadencia, regiones agrarias - subdivididas en re- 
giones agrarias con producción predominantemente mediterránea, regiones de montaña y otras regiones agrarias desfavorecidas-, regiones urbanas con problemas especificos, regiones periféricas, entre las que incluye a las regiones insulares, y las regiones fronterizas (Comisión CEE, 1987, pág. 50).

En sintesis, esta tipologia regional propuesta por la Comisión podría quedar englobada en dos grandes grupos. Por una parte, aquellas regiones caracterizadas por una densidad de población baja, un grado de industrialización inferior a la media comunitaria, una fuerte dependencia del sector agrario, un indice de paro superior a la media, una escasa productividad laboral y una renta inferior a la media; son las regiones más atrasadas de la Comunidad, periféricas y fundamentalmente agrarias, localizadas en su mayor parte en el sector meridional y occidental de la Comunidad. Por otra parte, existen otras regiones que se enfrentan a problemas de reajuste estructural graves y que, por ello, presentan también altos índices de paro y unos signos evidentes de debilidad de la renta; se trata de antiguas regiones industriales, hoy día en declive, que desde un punto de vista geográfico presentan una menor concentración que las regiones atrasadas, encontrándose dispersas y situadas principalmente en la mitad Norte de la Comunidad.

Como hemos visto, el análisis de las diferencias regionales intracomunitarias de tipo socioeconómico pone de manifiesto que las sucesivas ampliaciones de la Comunidad han incrementado extremadamente las diferencias existentes entre las regiones, diferencias que pueden agravarse en los próximos diez años en razón de perspectivas demográficas muy diferentes según las regiones. Por todo ello se hace imprescindible la puesta en marcha de una verdadera política regional comunitaria, en pos de corregir unos desequilibrios regionales en continuo aumento.

Ya en el Preámbulo del Tratado de Roma, aunque no de forma explícita, se intuía la necesidad de una política regional, al reconocer formalmente la existencia de zonas desfavorecidas dentro de la Comunidad. Expresamente, en el párrafo quinto del Preámbulo se lee: “Preocupados por reforzar la unidad de sus economías y asegurar el desarrollo armónico, reduciendo las diferencias entre las diversas regiones y el retraso de las menos favorecidas..."'.

En el período comprendido entre 1957 y 1974 la Comunidad utilizó recursos financieros específicos para fomentar las políticas regionales na-

\footnotetext{
' Véanse otros artículos del Tratado CEE: $2 ; 39,2$ a); 49d); 75; 82; 92,2.c) y 3 a) y c); 130.
} 
cionales. Estas intervenciones financieras se destinaron fundamentalmente unas en favor de la reconversión de las regiones carboníferas y siderúrgicas a través de la CECA, otras en favor de la reestructuración de las zonas agricolas por medio de la sección "Orientación" del FEOGA, y por último, una buena parte de los recursos se destinó en favor de la reeducación profesional y la reinstalación de los trabajadores, a cargo de la CECA y del Fondo Social Europeo.

Sin embargo, hasta la década de los setenta no nace una Política Regional propia de la Comunidad, concebida «con el fin de aumentar el grado de convergencia entre los Estados miembros y garantizar un mejor reparto de las actividades económicas sobre todo el territorio comunitario mediante la reducción de las disparidades regionales en materia de empleo y de logros económicos" (Comisión CEE, 1985, pág. 20). En reacción a la crisis que afecta de diferente manera a los Estados miembros desde 1973, éstos siguen a menudo políticas contradictorias desde el punto de vista comunitario, y el progresivo deterioro de la situación actual acentúa estas incompatibilidades (BUZELAY, 1982, pág. 336). Por ello uno de los principales objetivos que tiene la política regional europea es la coordinación de las políticas regionales de los Estados miembros. En este sentido, «la política regional debe encargarse de asegurar que la convergencia real entre los Estados miembros beneficie, ante todo, a las regiones problemáticas, y para ello debe orientarse en dos direcciones: en primer lugar, debe procurar que en las regiones menos desarrolladas la producción, el empleo y las inversiones se desarrollen con una dinámica superior a la de la tendencia general; en segundo lugar, debe procurar que las regiones industriales con problemas de reajuste hagan frente a los inevitables procesos de adaptación estructural sin atrofiar o estancar la economia regional»» (Comisión CEE, 1987, pág. VI).

De acuerdo con ello, se creó el FONDO EUROPEO DE DESARROLLO REGIONAL (FEDER), que fue instituido por el Reglamento del Consejo n. ${ }^{\circ} 724 / 725$ de 18 de marzo de 1975. La función del FEDER, señala el artículo 1 , consiste en corregir los principales desajustes regionales que, dentro de la Comunidad, proceden de la preponderancia agricola, el cambio industrial y el desempleo estructural.

En este Reglamento ya se preveía que las solicitudes de ayuda deberian canalizarse a través de los Programas de Desarrollo Regional (PDR) que cada Estado transmitiría a la Comisión. La tarea de especificar el contenido de estos programas fue atribuida a los Estados miembros, si bien se han de elaborar de conformidad con un esquema común establecido por el Comité de Política Regional, creado por la decisión del Consejo 185/75, publicada 
el 21 de mayo de 1975. Desde esta perspectiva, los PDR constituyen un importante instrumento de coordinación de las diferentes políticas regionales de los Estados miembros, así como el marco de referencia para acogerse a las ayudas del FEDER.

La dotación presupuestaria del FEDER, como dispone el art. 2, es fijada anualmente dentro del Presupuesto General de las Comunidades Europeas. La tabla adjunta indica la cuantia de los recursos apropiados por el FEDER y el porcentaje sobre el Presupuesto Total, según los distintos años. Como podemos observar, desde su creación en 1975 su volumen ha pasado del 4.8 por 100 del presupuesto general de la CEE al 8.6 por 100 en 1986.

DOTACION PRESUPUESTARIA DEL FEDER 1975-1986

\begin{tabular}{|c|c|c|c|}
\hline AÑO & $\begin{array}{c}\text { CANTIDADES } \\
\text { (MILL. ECUS) }\end{array}$ & $\begin{array}{c}\text { INCREMENTO } \\
\text { ANUAL EN \% }\end{array}$ & $\begin{array}{c}\text { \% DE PARTICIPACION EN } \\
\text { EL PRESUPUESTO CEE }\end{array}$ \\
\hline 1975 & 257,6 & - & 4,8 \\
1976 & 394,3 & 53,1 & 5,6 \\
1977 & 378,5 & $-4,0$ & 4,9 \\
1978 & 581,0 & 53,5 & 4,6 \\
1979 & 945,0 & 62,7 & 6,1 \\
1980 & $1.165,0$ & 23,3 & 6,7 \\
1981 & $1.540,0$ & 32,2 & 7,3 \\
1982 & $1.759,5$ & 14,3 & 7,6 \\
1983 & $2.010,0$ & 14,2 & 7,6 \\
1984 & $2.140,0$ & 6,5 & 7,3 \\
1985 & $2.289,9$ & 7,0 & 8,5 \\
1986 & $3.098,0$ & 35,3 & \\
\hline
\end{tabular}

Fuente: Fondo Europeo de Desarrollo Regional XII. El FEDER en cifras (1986), pág. 2.

Durante el periodo 1975-1984 el sistema de distribución de los recursos del FEDER fue el de cuotas fijas para cada país, pero a consecuencia del nuevo reglamento comunitario, que entró en vigor el 1 de enero de 1985, se implantó el sistema de horquillas, o bandas indicativas de participación, con un límite inferior y superior ya definidos. La distribución de estas ayudas muestra una gran concentración en algunos paises, que son los que preci- 
samente evidencian los desequilibrios regionales más acusados. Así, la periferia meridional y occidental de la Comunidad (Grecia, Italia, España, Portugal, Irlanda y Reino Unido) es la más beneficiada por los recursos del FEDER, mientras que los paises centrales reciben porcentajes mucho menores.

Los recursos del FEDER fundamentalmente se invierten en: infraestructuras, que concentran más del 80 por 100 de las inversiones; industria, artesanado y servicios; y estudios (ayudas destinadas a facilitar el asesoramiento en materia de comercialización, gestión e innovación). Estas intervenciones del FEDER adoptan la forma de programas y proyectos de inversión o de estudios. A raiz del nuevo Reglamento, la financiación mediante programas se ha incrementado más que la de los proyectos. Así, está previsto que en plazo de tres años se destine a programas el 20 por 100 de los recursos del Fondo. En este sentido, el aumento de los programas para intervenciones de carácter regional ha supuesto uno de los aspectos de mayor novedad.

Así pues, el FEDER participa en la financiación de:

- Programas comunitarios, puestos en marcha por iniciativa de la Comisión, que tras el estudio de unos programas elaborados por los Estados miembros interesados los aprueba y financia. En la actualidad ya están en marcha los dos primeros programas comunitarios: Star (para un mejor acceso a los servicios avanzados de telecomunicaciones) y Valoren (valorización del potencial energético endógeno) (XX Informe General CEE, 1986, pág. 223). Próximamente entrarán en vigor dos nuevos programas comunitarios que deberán ser cofinanciados por el FEDER como ayuda a las regiones afectadas por la reestructuración de la construcción naval (Renaval) y de la siderurgía (Resider) (XXI Informe General CEE, 1987, pág. 205). De este modo se pretende responder al objetivo de reconversión de las regiones industriales en crisis a través de políticas estructurales comunitarias.

- Programas nacionales de interés comunitario, puestos en marcha por iniciativa del Estado miembro y adoptados de común acuerdo con la Comisión.

- Acciones encaminadas a revalorizar el potencial de desarrollo endógeno de las regiones, mediante la ayuda a pequeñas y medianas empresas, con el fin de fomentar las posibilidades de desarrollo interno de cada región. 
- Acciones comunitarias integradas de desarrollo que pueden tener prioridad en la gestión de los recursos del FEDER (art. 34 del Reglamento). Estas operaciones están constituidas por un conjunto de inversiones referidas a un área geográfica concreta afectada por graves problemas que limitan su desarrollo. Cabría destacar en este apartado los PIM (Programas Integrados Mediterráneos), aprobado su Reglamento el 23 de julio de 1985.

Por último, además de los programas, el FEDER también financia:

- Proyectos de inversiones en infraestructura (que en 1986 absorbieron el 87 por 100 de las ayudas concedidas) o en actividades industriales, artesanales y de servicios.

- Estudios que estén estrechamente vinculados a las operaciones del FEDER, o que tengan un especial interés para una mejor utilización de los fondos.

Respecto a la localización de las regiones que reciben más ayuda del FEDER, éstas cubren la mayor parte del sector meridional y occidental de la Comunidad, además de alguna otra región aislada; en total, a comienzos de 1987, abarcan aproximadamente un tercio de la superficie comunitaria y en ellas viven 132,4 millones de habitantes, es decir, el 41.3 por 100 de la población total de la Comunidad. Sin embargo, la importancia relativa de estas regiones asistidas es muy diferente de unos Estados a otros; así, por ejemplo, estas regiones concentran alrededor del 15 por 100 de la población en los Paises Bajos, entre el 24 y 48 por 100 en Dinamarca, Bélgica, Alemania, Reino Unido, Francia, Italia y España, el 66 por 100 en Grecia y un 80 por 100 en Luxemburgo y Portugal; Irlanda es para el FEDER elegible en su totalidad ${ }^{2}$. En cuanto al porcentaje de superficie que ocupan estas regiones dentro de cada Estado, todas las regiones acaparan más del 40 por 100 de la superficie nacional, con la excepción de los Países Bajos, cuyas regiones elegibles para las ayudas del FEDER afectan a un 20 por 100 de su superficie, y el caso de Irlanda, ya antes apuntado, en el que todo su territorio es elegible para el FEDER.

En definitiva, 30 años después de la creación de la Comunidad Económica Europea, el progresivo aumento de los desequilibrios regionales refleja la he-

${ }^{2}$ Fondo Europeo de Desarrollo Regional, XII Informe anual. 
terogeneidad existente entre los Estados miembros, asi como las disparidades internas de los propios Estados. Esta realidad contrasta vivamente con el objetivo de igualdad y armonia en el desarrollo que se había propuesto la política regional comunitaria. Así pues, la Comunidad sigue necesitando con urgencia una política regional ambiciosa en sus medios y comunitaria en su aplicación. El futuro nos dirá si la Comunidad ha tenido la voluntad y la capacidad de afrontar el desafio que ella misma se ha impuesto ${ }^{3}$.

\section{EL NUEVO MARCO DE LA POLITICA TERRITORIAL}

La nueva coyuntura española está marcada por un conjunto de circunstancias que van a condicionar - de hecho ya lo están haciendo en el presente - el futuro funcionamiento de su política regional. Estos hechos fundamentales serian:

1) La nueva situación de crisis económica.

2) La estructuración territorial del Estado Autonómico como consecuencia del desarrollo de la Constitución de 1978.

3) La integración de nuestro país en la CEE.

Estas tres realidades hacen ineludible el replanteamiento de la política regional sobre nuevas bases que tengan presente:

- La existencia de una nueva problemática regional, alejada de la tradicional dicotomía que dividía a las regiones en rurales y retrasadas (subdesarrolladas) y urbanas. La crisis económica ha alterado sustancialmente el mapa de las regiones-problema. "Junto a la debilidad tradicional de ciertas áreas como las zonas de montaña y las zonas rurales, la crisis nos ha traido nuevos hechos como la desindustrialización en las áreas desarrolladas, la

${ }^{3}$ Cf. P. ROMUS, (1982): “La politique régionale des communautés européennes", en Les politiques régionales en Europe. Actes du Colloque de Genève. Collection L.E.P., Lausanne. Pag. 312. 
iniciación de los procesos de desuburbanización, principalmente en las grandes ciudades, la relocalización progresiva de la estructura productiva (industria y servicios) en el territorio y la degradación de algunas zonas, principalmente en las áreas turísticas" (A. VAZQUEZ BARQUERO, 1985).

- La construcción del Estado de las Autonomias ha abierto un proceso de grandes expectativas en nuestro pais. La posibilidad de hacer frente a los problemas territoriales de forma descentralizada ha modificado el planteamiento centralista de solucionar estos problemas por la via de la imposición, "de arriba a abajo». El nuevo camino abierto, participativo y democrático, resulta más atractivo y positivo pero plantea algunos interrogantes que deberán, en buena lógica, irse despejando a medida que el nuevo Estado vaya consolidándose.

La nueva estructuración del espacio delimita, al menos, cuatro niveles de poder territorial que es necesario coordinar si se quiere definir coherentemente una política regional. Son estos: el nivel municipal, el autonómico, el estatal y el supraestatal de la Comunidad Europea. La necesidad de imbricar entre si estos cuatro escalones del territorio se hace aún más imprescindible si tenemos en cuenta, que el poder de toma de decisión en cada uno de ellos puede recaer en grupos políticos ideológicamente poco afines e incluso enfrentados entre sí.

A esta realidad que no tiene por qué considerarse como negativa, aunque sí al menos conflictiva, debe unirse la circunstancia histórica en que nos encontramos. La larga inercia de un Estado fuertemente centralizado, como el nuestro, ha creado hábitos y costumbres que no desaparecen de la noche a la mañana. Es desde esta perspectiva como pueden comprenderse los recelos de las administraciones periféricas, difíciles de disipar, al menos en un plazo prudencialmente corto de tiempo.

- Por último, la reciente integración española en la CEE nos obliga, para tener acceso a los fondos distribuidos por el FEDER, a la coordinación con el resto de los paises comunitarios en materia regional, de acuerdo a una estructura común.

Este esquema de funcionamiento es imperativo en la necesidad de elaboración por todos los paises miembros de unos Programas de Desarrollo Regional (PDR), como instrumentos técnicos capaces de analizar la problemática regional desde una perspectiva integradora. 
En resumen, que las nuevas circunstancias analizadas han modificado sensiblemente los presupuestos básicos en los que se asentaba nuestra tradicional política regional. Esta política -como cualquier otra- debe dar respuesta adecuada a los siguientes interrogantes: 1) Qué problemas hay que resolver, o dicho de otro modo, hacia qué objetivos deben dirigirse los esfuerzos niveladores del territorio. 2) Qué instrumentos técnicos y financieros van a ser utilizados en la búsqueda de su solución. 3) Cuáles van a ser las instituciones que intervengan y gestionen los programas de actuación definidos.

Pues bien, estos tres interrogantes encuentran respuesta en el nuevo marco determinado por la situación económica y política por la que atraviesa nuestro pais. En primer lugar, la problemática regional se ha diversificado con la crisis económica. Es decir, que hay que hacer frente a nuevos problemas territoriales. En segundo lugar, la integración a Europa ha definido unos nuevos instrumentos de actuación regional. EI PDR se ha convertido así, "en un instrumento básico para conseguir una coordinación entre los distintos niveles de gobierno, autonómico, nacional y comunitario" según expresa la metodología para su elaboración aprobada el 25 de marzo de 1985. Por último, la estructuración del Estado Autonómico ha delimitado unos nuevos gestores: las Comunidades Autónomas, que junto con el Gobierno de la nación, deben actuar dentro del marco constitucional.

Analizados, en las dos primeras partes de esta ponencia, los principales problemas territoriales que afectan a nuestro país en el momento inicial, así como los principales instrumentos financieros empleados por la CEE en la consecución de su política regional, vamos a centrar nuestro análisis en las perspectivas que se abren con la nueva figura del planeamiento regional en contraste con la anterior política territorial, que podemos denominar como "clásica", valorándola a la luz de los resultados obtenidos en el corto espacio de tiempo transcurrido desde su implantación, así como de la problemática específica de la nueva división territorial de nuestro país.

\section{EL CAMBIO DE LA POLITICA REGIONAL}

Tradicionalmente, la política española en materia regional ha utilizado los incentivos como instrumento de actuación. Sus origenes se remontan, aunque puedan existir acciones puntuales anteriores, a comienzos de los años sesenta, 
y más en concreto a los Planes de Desarrollo. El primero de ellos, aprobado a finales del año 1963, contenía como soporte básico la política de los Polos de Desarrollo, dirigida a incentivar el desarrollo de áreas puntuales. El concepto se amplió en el tercero de los planes, cuya promulgación, en el año 1972, pretendía la creación de las denominadas Grandes Areas de Expansión Industrial (GAEI), zonas geográficamente más extensas, aunque predominase igualmente la filosofía del sistema de incentivos para estimular la localización industrial en zonas determinadas. También durante el comienzo de la década de los años sesenta se promulgó la Ley de Industrias de Interés Preferente, sobre la cual han cristalizado las principales figuras de incentivación de actividades directamente productivas: Zonas de Preferente Localización Industrial (ZPLI), Zonas de Preferente Localización Industrial y Minera (ZPLIM), Zonas de Preferente Localización Industrial Agraria (ZPLIA) y Poligonos de Preferente Localización Industrial.

Aún, hoy en día, continúa actuándose en determinadas áreas del territorio nacional de manera similar. Piénsese en las Zonas de Urgente Reindustrialización (ZUR), surgidas tras la aprobación de la Ley de 26 de julio de 1984 sobre Reconversión e Industrialización. En este caso, se trata de hacer frente en determinadas áreas, especialmente afectadas por la crisis (Asturias, Cádiz, Vigo, Ferrol, Bilbao, Madrid y Barcelona), a los efectos negativos consecuentes a los procesos de declive industrial. Frente a problemas nuevos se aplican los métodos de siempre. En esencia, se pretende influenciar la localización de la industria y otras actividades económicas en áreas deprimidas y ello mediante incentivos fiscales y financieros, como subvenciones y bonificaciones fiscales, bonificaciones en tipos de interés, creditos oficiales preferentes... etc.

Esta política de incentivos, que ha tenido un cierto éxito en épocas de expansión económica, ha fracasado, tanto por su carácter "arcaico, indiscriminado y profuso» (G. SAENZ DE BURUAGA, 1985) como por carecer de una coordinación con las políticas macroeconómicas y sectoriales de carácter más general, lo que ha supuesto, en muchas ocasiones, un despilfarro de recursos en un pais como el nuestro, no muy sobrado por cierto de ellos.

Hoy en día, el enfoque de una política regional basada fundamentalmente en el establecimiento de incentivos para fomentar las inversiones en las áreas deprimidas, ofrecería, además, notorias contradicciones con las directrices emanadas de la propia CEE. 
La transformación de la política regional en el nuevo marco de la división autonómica y de la integración a Europa se basa en un nuevo proceso en el que cambia el diseño, la formulación y el seguimiento de la misma. Veamos de qué manera.

Si bien es cierto, que con anterioridad a la puesta en marcha de los nuevos instrumentos de planificación territorial, existieron otros de elaboración similar, como los Planes Directores Territoriales de Coordinación (PDTC) o los Programas Económicos Regionales (PER) su vida resultó tan efímera que "seguramente ni aquéllos ni éstos pasarán a la crónica de la historia" (L. LAZARO ARAUJO, 1986). No es, por tanto, hasta mediados de los años ochenta, cuando puede considerarse que estamos ante la presencia de una nueva situación que va a permitir la elaboración de una política territorial sobre nuevas pautas. Por su importancia transcendental, merece que la dediquemos un apartado diferenciado.

\section{EL PROGRAMA DE DESARROLLO REGIONAL, INSTRUMENTO BASE DE LA PLANIFICACION TERRITORIAL}

El éxito o fracaso de una política regional que pretenda reducir los desequilibrios territoriales de un área determinada, depende de dos elementos fundamentales:

- La existencia de fondos económicos o inversiones destinados a ello.

- La elaboración de un plan y de unos programas coherentes que sean gestionados racionalmente por las administraciones públicas.

Desde un punto de vista financiero, las inversiones tendentes a solucionar los problemas territoriales proceden de diversas fuentes, pero existen dos fundamentales: el FEDER y el Fondo de Compensación Interterritorial $(\mathrm{FCl})$. El primero ya ha sido analizado en la segunda parte de esta ponencia por lo que no merece la pena detenerse excesivamente en su exposición. Por su parte, el $\mathrm{FCl}$ debía ser creado, según señala el artículo 158.2 de la Constitución, "con el fin de corregir los desequilibrios interterritoriales y hacer efectivo el principio de solidaridad". La Ley Orgánica de Financiación de las Comunidades Autónomas (LOFCA) de septiembre de 1980 y posteriormente la Ley 
del Fondo de Compensación Interterritorial (LFCI) de mayo de 1984, terminaron de configurar este instrumento de desarrollo regional, con la finalidad de perseguir una distribución más justa de la renta y de la riqueza. La cuantía del fondo fue definida según ley en torno al 30 por 100 de la inversión pública de cada ejercicio y los criterios de reparto entre las Comunidades Autónomas más desfavorecidas se han establecido en función del inverso de la renta por habitante, la tasa de población emigrada, el porcentaje de desempleo y la superficie territorial. Sin embargo, y con ser importantes, estos recursos procedentes del exterior de cada Comunidad Autónoma son aún reducidos para hacer frente a la gran cantidad de problemas que deben acometerse.

Pero quizás, la novedad más importante de la nueva política regional reside en los nuevos instrumentos de coordinación: los PDR. La importancia de este instrumento de planificación se deriva de su capacidad para coordinar la política regional, no sólo dentro de un país a escala intranacional, sino entre los diversos países que integran la Comunidad Europea. La propia CEE ha definido una estructura común de los programas que afectan a todos sus miembros integrantes. En nuestro país, cada Comunidad Autónoma debe encargarse de su elaboración en un modelo de planificación descentralizada, donde el Estado central se reserva la facultad de la coordinación y la aprobación de la metodología común. Esta manera de afrontar la problemática económico-regional contrasta con la tradicional, en donde «primero se dispondría de un plan nacional y luego ese plan de disgregaría en planes regionales. Es decir, un modelo de planificación regional desde arriba. En cambio, la Constitución española apunta a un modelo de planificación desde abajo, desde las Comunidades Autónomas, con un criterio mucho más participativo y democrático» (L. LÁZARO ARAUJO, 1986).

Los PDR se han estructurado en cinco capitulos que dan respuesta a las diferentes fases que deben integrar una política territorial bien estructurada: conocer los problemas del territorio, definir los principales objetivos de desarrollo, delimitar las acciones a ejercer, asi como los recursos financieros a emplear y por último, realizar un plan de ejecución y seguimiento.

El primer capítulo lleva por título «Análisis Económico-Social» y su objetivo fundamental, según la propia metodologia aprobada por el Gobierno central es "realizar un análisis económico de la evolución y situación de la región con el fin de detectar los principales desequilibrios que padece y sus causas". Se trata, no tanto de una mera descripción estadistica, sino de realizar un diagnóstico analítico de los problemas regionales y sus causas. El capítulo se divide en varios apartados de tipo sectorial y geográfico, donde deben ser analizados aspectos relativos a la población, la producción, el 
empleo y la renta como elementos fundamentales de la estructura económica, y la infraestructura económica y estructura urbana como aspectos relevantes de la organización territorial. Finalmente, el resto de los epigrafes se ocupan del estudio de los principales desequilibrios que padece la región y sus causas, el impacto de las acciones iniciadas en el pasado para remediar estos desequilibrios y, sobre todo, las posibilidades de desarrollo de la región incluidos los estrangulamientos.

El segundo capítulo propone especificar, sobre la base de los problemas detectados en el capitulo anterior, los objetivos que pretenden alcanzarse en la región en el período de la planificación. Estos objetivos deben, lógicamente, ser coherentes con los objetivos macroeconómicos generales y sectoriales a nivel de la nación y de la propia CEE, aconsejándose la inclusión de un calendario de realización de los mismos, año a año. Los objetivos, considerados como esenciales, se refieren a la creación de empleo y de infraestructuras, pudiendo existir otros de carácter demográfico, de modificación de la estructura productiva... etc.

El tercer capítulo es relativo a las acciones de desarrollo que se van a poner en práctica para el logro de los objetivos señalados en el capítulo anterior. Las medidas adoptadas se clasifican en dos grupos: aquellas que van dirigidas directamente al territorio regional y aquellas otras de política nacional, general o sectorial, con un impacto significativo sobre el desarrollo de la región. Se aconseja en la elaboración "que tales medidas estén periodificadas, incluyendo desfases temporales en su efectividad" y se refieren, a subvenciones, a la inversión o al empleo, crédito oficial, bonificaciones o exenciones fiscales, ... etc.

El cuarto capítulo pretende evaluar los recursos que se van a destinar a financiar las medidas contenidas en el capítulo anterior, desglosándose por fuentes de financiación: local, regional, estatal o procedentes de la CEE, asi como por el tipo de gastos.

Por último, en el capítulo quinto se señalan los organismos responsables de la ejecución de cada parte del programa con especificación de las tareas asignadas a cada uno de ellos.

En general la experiencia puesta en marcha debe valorarse positivamente y ello por dos motivos fundamentales:

1) Porque ha supuesto el comienzo de un proceso de programación regional que no tiene antecedentes en nuestro pais (salvo, quizás, las ex- 
cepciones comentadas en los PDCT o los PER), ni en lo relativo a la manera de enfocar los problemas: globalmente, ni en la forma participativa de coordinar el trabajo entre las Comunidades Autónomas y la Administración Central.

2) Porque los PDR están sirviendo para profundizar notablemente en el conocimiento, análisis y diagnóstico de los problemas específicos que en materia de desequilibrios regionales afectan a cada territorio autonómico.

Sin embargo, es necesario señalar la existencia de algunos interrogantes, derivados, tanto de la falta de experiencia en la planificación regional bajo unos presupuestos como los analizados anteriormente, como por el lento desarrollo del Estado de las Autonomías, que está provocando problemas de engarce entre las diferentes instancias de poder territorial. Algunos de estos problemas ya están surgiendo a la luz, a pesar del corto espacio de tiempo transcurrido desde su puesta en funcionamiento.

Ciñéndonos, de primeras, al contenido de los PDR ya elaborados, éstos presentan algunas deficiencias que es necesario señalar. Analizando detenidamente la estructura de estos instrumentos de planificación se podría concluir que nos encontramos en la presencia de una «política regional auténtica, en el marco de la política económica global, habiéndose hallado la estrategia idónea capaz de permitir atender al logro de los objetivos que le son propios a la política de desarrollo regional, sin interferir en la consecución de los restantes objetivos macroeconómicos. Sin embargo si no se actúa de forma rigurosa, determinando correctamente las potencialidades futuras de cada una de las Comunidades Autónomas, y selectivamente sobre los principales estrangulamientos de sus respectivas economias, dado que los recursos para la inversión son escasos, se correría el riesgo de convertir a estos PDR en documentos carentes de operatividad, como les ha ocurrido a algunos otros planes regionales que les precedieron" (J. HERNÁNDEZ ARMENTEROS y J. VILLENA PEÑA, 1986).

\section{Pasando al análisis de detalle, podemos señalar:}

- La información, contenida en el capítulo primero, ha sido positivamente valorada por la Comisión de Política Regional de la CEE, aunque las estadisticas utilizadas hacen referencia a periodos temporales atrasados que se extrapolan tendencialmente. Además, no todas las Comunidades Autónomas han elaborado su información de manera semejante, lo que conlleva una relativa falta de homogeneidad. 
- Relativo a los objetivos de desarrollo, la propia metodología elaborada por el Gobierno central, adelantándose a las posibles dificultades que se vislumbraban en su concreción, ya adelantaba las siguientes observaciones: a) que no fueran enunciados en términos muy generales, aconsejando su cuantificación, $b$ ) que no constituyeran un inventario de necesidades o deseos, sino que debian ser realizables, $c$ ) que incluyeran un calendario de compromiso temporal, y d) que debian insertarse en un marco económico y social más amplio, teniendo en cuenta los objetivos macroeconómicos nacionales y de la propia CEE.

El resultado ha sido, sin embargo, que no siempre se han tenido presentes estos criterios y que se echa en falta un encadenamiento lógico entre el diagnóstico de la situación, los objetivos que se proponen y las acciones a poner en práctica para conseguirlos.

- Por último, y relativo a los recursos financieros, ha existido en los planificadores una excesiva generosidad en la enumeración de los objetivos, lo que contrasta con las limitaciones de los presupuestos.

A nivel más general se adivinan otro cúmulo de problemas e interrogantes de, quizás, más difícil solución. En este sentido, el PDR ha sido concebido como un mecanismo de coordinación horizontal de cada Comunidad Autónoma con el resto, así como de coordinación vertical, bien en sentido ascendente con la Administración Central y la propia CEE, bien en sentido descendente con los gobiernos municipales. Sin embargo, preocupa el hecho de que mientras se han especificado, con mayor o menor amplitud, los objetivos, que pretenden alcanzar los poderes públicos regionales en su ámbito territorial, no estén elaborados los de la política regional a escala nacional, es decir los que desarrolla y ejecuta la Administración Central. Existe el peligro, muy real, de que los PDR aprobados no sirvan de instrumentos coordinadores de la política territorial de las Comunidades Autónomas y el Estado.

Además, «las reservas ya apuntadas entre la Administración Central y Autonómica se reproducen entre los gobiernos autonómicos y los de las Corporaciones Locales de su ámbito territorial. La consecuencia es que una parte no despreciable de la actividad financiera del sector público directamente relacionada con el desarrollo regional de base local no queda reflejada en el PDR» (L. LAZARO ARAUJO, 1986). 


\section{CONCLUSIONES}

Con los aspectos positivos y negativos señalados, parece que la incorporación de España a la CEE ha abierto el camino a la elaboración de una política regional coherente con las nuevas coordenadas de nuestro ordenamiento jurídico.

Quizás, las mayores dificultades surjan de la propia concepción que subyace en toda política de este tipo, aplicada en un país capitalista, de libre iniciativa, como España, donde la competencia individual y colectiva es connatural con la desigualdad económica de los individuos y de los territorios. El propio texto constitutivo de la CEE, señala en su artículo 92 que «las ayudas destinadas a favorecer el desarrollo económico de regiones en las que el nivel de vida sea anormalmente bajo o en las que exista una grave situación de subempleo, podrán considerarse compatibles con el Mercado Común". La propia frase es un reconocimiento de que de los dos principios que tratan de hacerse compatibles: el de solidaridad y el de libertad de iniciativa, el último prima sobre el primero. Parece, por tanto, que una política de intervención pública, que trate de disminuir los desequilibrios creados por un sistema competitivo, debe dirigirse, exclusivamente, a amortiguar los efectos más negativos del sistema. No extraña, en consecuencia, que los fondos públicos destinados a financiar operaciones de esta índole sean limitados y no alcancen a sufragar sino una parte reducida de las necesidades existentes.

Sin embargo, y aún siendo evidentes los condicionamientos de carácter general que el funcionamiento del sistema económico encierra, la nueva politica territorial emprendida posee caracteres de una mayor racionalidad en la gestión, que contrasta con anteriores etapas históricas. En primer lugar, es la primera vez que la planificación regional adquiere una perspectiva global en la delimitación de los problemas y de sus soluciones, pretendiendo hacer congruentes los objetivos a escala regional y nacional. $Y$, en segundo lugar, la nueva política se pretende llevar a efecto de forma participativa y democrática. Esta nueva orientación de la problemática territorial permite, al menos de momento, encarar el futuro con un cierto margen de confianza y optimismo. 


\section{BIBLIOGRAFIA}

CASTELL, M. (1985). Metrópolis, territorio y crisis. Asamblea de Madrid.

CONDOMinES, J. (1982): «Les sept Europes: une typologie des régions de la Communauté Européenne», en Les politiques régionales en Europe, Actes du Colloque de Genève. Collection L.E.P. Lausanne.

Fondo Europeo de Desarrollo Regional. XI Informe Anual (1985). Comisión de las Comunidades Europeas.

Fondo Europeo de Desarrollo Regional. XII Informe anual (1986). Comisión de las Comunidades Europeas.

García Alvarez, A. (1981): “Bases para una política de acción territorial», Estudios Territoriales, 4, págs. 73-120.

Hernández Armenteros, J. y Villena Peña, J. (1986): «Una valoración de la coordinación y coherencia de los objetivos de los P.D.R.s", Jornadas sobre Programas de Desarrollo Regional. Barcelona, 16 págs.

Lázaro Araujo, L. (1986): “Los Programas de Desarrollo Regional y la experiencia española de planificación regional entre 1975 y 1985", Jornadas sobre Programas de Desarrollo Regional. Barcelona, 51 págs.

Programa de Desarrollo Regional. España (1986). 2 vols.

Las regiones de Europa (1985). Segundo informe periódico sobre la situación socioeconómica de las regiones de la Comunidad. 
Las regiones de la Comunidad ampliada (1987). Tercer informe periódico por la Comisión sobre la situación y evolución socioeconómica de las regiones de la Comunidad.

Rodriguez Rodríguez, V. (1986): “Los Programas de Desarrollo Regional en España", IV Coloquio Ibérico de Geografía. Coimbra, págs. 379-392.

SAENZ de BuRuaga, G. (1985): "La planificación nacional en la España de las Autonomias", Actas de la X Reunión de Estudios Regionales, t. II, Valladolid, págs. 553-582.

Vázquez BARQUero, A. (1985): «Hacia un nuevo enfoque de la política regional de España", Actas de la $X$ Reunión de Estudios Regionales, t. II, Valladolid, págs. 639-698.

20. Informe General CEE (1986). Sección 10. Política Regional. Pp. 223-231.

21. Informe Genera/ CEE (1987). Sección 9. Política Regional. Pp. 205-213. 